\title{
DISARTRIA NA DOENÇA DE WILSON: ANÁLISE DE DOIS CASOS EM FASES DISTINTAS
}

\section{Dysarthria in Wilson's disease: analysis of two cases in different stages}

\author{
Natália Casagrande Brabo ${ }^{(1)}$, Maysa Luchesi Cera ${ }^{(2)}$, Simone dos Santos Barreto ${ }^{(3)}$, Karin Zazo Ortiz ${ }^{(4)}$
}

\begin{abstract}
RESUMO
Tema: disartria na Doença de Wilson. Procedimentos: estudo de caso de dois pacientes com o diagnóstico da doença, ambos adultos jovens, do sexo feminino e com seis anos de escolaridade. Quatro fonoaudiólogas realizaram a avaliação perceptivo-auditiva de modo independente, na qual foram investigados os seguintes parâmetros: tipo de voz, loudness, pitch, ataque vocal, instabilidade fonatória e ressonância. O grau de alteração de cada parâmetro foi mensurado através de uma escala analógico-visual. Além disso, foram obtidas medidas de inteligibilidade por transcrição ortográfica. A análise acústica foi realizada por meio do programa Praat, com a obtenção de medidas de frequência fundamental, de perturbação e ruído e medidas temporais. Resultados: a fala de ambos os pacientes foi caracterizada por: redução do tempo máximo de fonação, voz soprosa e tensa, ataques vocais aspirados, instabilidade vocal, aumento de shimmer e proporção ruído-harmônico, hipernasalidade, redução da diadococinesia oral, da inteligibilidade e da velocidade da fala. Os resultados obtidos sugeriram que o paciente que cursa com a Doença de Wilson há mais tempo, apresentou prejuízo mais acentuado na maioria dos parâmetros perceptuais e acústicos analisados. Conclusão: foram identificadas manifestações comuns entre os casos, as quais podem constituir-se em traços característicos da fala de pacientes disártricos com Doença de Wilson. Todavia, diferenças também foram evidenciadas, as quais podem estar relacionadas ao tempo de evolução da doença.
\end{abstract}

DESCRITORES: Disartria; Degeneração Hepatolenticular; Medida da Produção da Fala; Acústica da Fala

\section{INTRODUÇÃO}

A Doença de Wilson (DW), também conhecida como degeneração hepatolenticular, é uma doença genética degenerativa, de herança autossômica

(1) Fonoaudióloga; Pesquisadora do Departamento de Fonoaudiologia da Universidade Federal de São Paulo, UNIFESP, São Paulo, SP; Especialização em Distúrbios da Comunicação Humana pela Universidade Federal de São Paulo.

(2) Fonoaudióloga; Pesquisadora do Departamento de Fonoaudiologia da Universidade Federal de São Paulo, UNIFESP, São Paulo, SP; Mestranda em Distúrbios da Comunicação Humana pela Universidade Federal de São Paulo.

(3) Fonoaudióloga da Prefeitura do Rio de Janeiro, Rio de Janeiro, RJ; Doutoranda em Distúrbios da Comunicação Humana pela Universidade Federal de São Paulo.

(4) Fonoaudióloga; Professora Adjunta do Departamento de Fonoaudiologia da Universidade Federal de São Paulo, UNIFESP, São Paulo, SP; Pós-doutorado em Neurociências pela Universidade Federal de São Paulo.

Conflito de interesses: inexistente recessiva, que associada a um distúrbio do metabolismo do cobre, resulta no acúmulo deste metal em órgãos como: fígado, cérebro e córneas ${ }^{1}$.

Caracteriza-se como uma doença de caráter progressivo e, caso não seja diagnosticada e tratada precocemente, pode ser fatal. Atualmente, o tratamento com zinco é considerado o mais eficaz, seguro e barato e visa à normalização da concentração de cobre livre na corrente sanguínea, para desintoxicação ${ }^{2,3}$.

Seus sinais e sintomas surgem, comumente, na adolescência e na idade adulta, no entanto, a doença também pode manifestar-se antes dos 6 anos ou após os 40 anos de idade. Os sinais neurológicos podem variar entre os pacientes, com a espasticidade mais proeminente do que a ataxia ou a rigidez em alguns casos, enquanto em outros, a distonia apresenta-se como a principal manifestação ${ }^{1}$. Apesar das diferenças entre os quadros, a disartria é conhecida como uma característica neurológica proeminente na DW, aparecendo como 
manifestação inicial em vários casos relatados na literatura ${ }^{4}$.

Sabe-se que a disartria é um nome coletivo para as alterações de fala resultantes de distúrbios no controle de seu mecanismo, devido a danos no sistema nervoso central e/ou periférico ${ }^{5}$. Alterações de fala podem causar comprometimento importante da inteligibilidade e, consequentemente, afetar a comunicação desses indivíduos. Como a DW tem caráter progressivo, as manifestações disártricas tendem a piorar, acompanhando a evolução do quadro geral do paciente.

O padrão perceptual dos sintomas de fala pode produzir informações com significância diagnóstica na avaliação de doenças neurológicas, assim como o inverso também é verdadeiro ${ }^{5}$. É possível encontrar, na literatura, estudos em que a alteração de fala foi empregada como critério para a classificação da gravidade da doença ${ }^{3}$. Nesse contexto, a avaliação fonoaudiológica pode mostrar-se um recurso importante tanto ao diagnóstico precoce quanto no acompanhamento desses pacientes.

Nos últimos anos, tem-se aplicada a análise acústica em sujeitos com doenças neurológicas. A análise perceptivo-auditiva é considerada padrão ouro para avaliação vocal e a inclusão da análise acústica pode fornecer importantes informações ${ }^{6}$. A análise acústica auxilia na quantificação dos dados e nas descrições das correlações dos julgamentos perceptivos de inteligibilidade de fala, qualidade vocal e tipo de disartria ${ }^{7,8}$.

Desta forma, os objetivos deste estudo foram caracterizar e comparar as manifestações de fala em dois pacientes com DW em fases de evolução distintas, por meio de análise perceptivo-auditiva e acústica da fala.

\section{APRESENTAÇÃO DOS CASOS}

Participaram desta pesquisa dois falantes disártricos com diagnóstico de DW, atendidos no Núcleo de Investigação e Intervenção Fonoaudiológica em Neuropsicolinguística da Universidade Federal de São Paulo (UNIFESP). Ambos os pacientes eram adultos jovens, do sexo feminino e possuíam seis anos de escolaridade. A seguir, serão apresentados a caracterização geral dos dois casos clínicos estudados:

Caso 1: paciente de 20 anos apresentou os primeiros sinais e sintomas e recebeu o diagnóstico da doença aos 17 anos, 3 anos antes da avaliação fonoaudiológica. A alteração da fala foi um dos primeiros sintomas da doença, assim como alterações de marcha e deglutição. Desde o diagnóstico, realizava tratamento medicamentoso com Penicilamina. Apresentou histórico familiar positivo: uma irmã diagnosticada com a doença. A avaliação fonoaudiológica revelou quadro de disartria hipocinética.

Caso 2: paciente de 30 anos com diagnóstico da doença desde os 21 anos de idade, 1 ano após o aparecimento dos primeiros sintomas. Manifestou inicialmente alterações de fala, postura e marcha, evoluindo cinco meses após com disfagia. Iniciou o tratamento medicamentoso com Penicilamina logo após o diagnóstico, quatro anos depois passou a utilizar Sulfato de Zinco. Também apresentou histórico familiar positivo: três irmãos, sendo dois já falecidos. Os resultados da avaliação fonoaudiológica indicaram a presença de disartria do tipo mista, com componentes flácido e espástico.

Os sujeitos foram submetidos à gravação de amostras da fala, captadas e armazenadas através do microfone Cyber Acoustics, modelo AC-100, acoplado ao notebook Toshiba, modelo L25, com o programa Sound Forge, Versão 4.5 (Sonic Foundry), em sua configuração padrão. A gravação foi realizada em ambiente silencioso, com o paciente sentado e o microfone posicionado a $5 \mathrm{~cm}$ de distância de sua boca.

As amostras gravadas foram obtidas através das seguintes tarefas de fala: emissão sustentada da vogal /a/ e das fricativas /s/ e /z/, contagem de números de 1 a 20 , diadococinesia oral de sílabas alternadas (/pa/, /ta/ e /ka/) e em sequência (/pataka/), repetição de listas de frases e palavras, leitura de texto e fala espontânea.

Para a avaliação perceptivo-auditiva, foram investigados os seguintes parâmetros: tipo de voz (soprosa, rouca, áspera, tensa ou sem desvio), loudness (aumentada, reduzida ou adequada), pitch (grave, agudo, adequado), ataque vocal (aspirado, brusco ou isocrônico), estabilidade vocal (instável ou estável) e ressonância (hipernasal, hiponasal, equilibrada). Nos parâmetros tipo de voz e ressonância, categorias adicionais puderam ser incluídas pelo avaliador, quando necessário. Além da classificação dos parâmetros, o grau de intensidade da alteração foi mensurado por meio da utilização de uma escala analógico-visual (EAV).

Quatro fonoaudiólogas realizaram a avaliação perceptivo-auditiva, de forma independente, a partir de amostras de fala editadas para cada paciente. Cada amostra continha um segmento central com 5 segundos de duração da emissão da vogal /a/, a sequência de números, duas frases com ocorrência distinta de fonemas nasais e um trecho de fala espontânea de 20 segundos. As avaliadoras foram solicitadas a ouvir as amostras de fala, quantas vezes julgassem necessárias, e classificar as vozes dos pacientes quanto aos parâmetros citados. Foram consideradas apenas as classificações que 
alcançaram resultados consensuais de, no mínimo, duas avaliadoras.

Para o julgamento da intensidade da alteração, uma linha de $100 \mathrm{~mm}$ de comprimento foi apresentada ao lado de cada parâmetro na folha de resposta, devendo a avaliadora, quando aplicável, marcar uma linha vertical que representasse o grau de alteração percebido. O grau de alteração na escala variava de modo crescente da esquerda para direita, com $0 \mathrm{~mm}$ representando a normalidade e $100 \mathrm{~mm}$ indicando alteração severa. As marcações na escala foram mensuradas em milímetros e foram calculadas as médias de cada parâmetro.

A análise perceptivo-auditiva foi complementada com a obtenção de medidas de inteligibilidade de frases e palavras. Dois ouvintes, não familiarizados com os estímulos e com a fala disártrica, foram solicitados a transcrever ortograficamente os estímulos gravados pelos falantes. As transcrições foram analisadas e pontuadas de acordo com sua correspondência em relação aos estímulos-alvo, sendo os escores de inteligibilidade calculados segundo a percentagem de sílabas corretamente transcritas em frases ou palavras.

A análise acústica foi realizada através do programa Praat versão 4.4.13, com a obtenção das seguintes medidas: freqüência fundamental (F0), variabilidade de F0, medidas de perturbação e ruído (jitter, shimmer, proporção ruído-harmônico - PRH) e medidas temporais (tempo máximo de fonação TMF, diadococinesia oral e velocidade da fala).

As medidas de frequência, de perturbação e ruído foram calculadas a partir de segmento de 3 segundos da porção medial da emissão da vogal sustentada /a/. Com a medida da F0 média, fornecida em $\mathrm{Hz}$, foi analisado o número de ciclos glóticos por segundo, enquanto sua variabilidade foi expressa pelo desvio-padrão de F0 (DP). Com as medidas de jitter e shimmer, dadas em percentagem, a variabilidade da frequência fundamental e da amplitude a curto prazo foram avaliadas, respectivamente. A proporção ruído harmônico $(\mathrm{PRH})$ mensurou a presença de ruído no sinal analisado, incluindo neste cálculo as contribuições da perturbação de amplitude e de freqüência.

Para a extração das medidas temporais, foram utilizados espectrogramas de banda larga, configurados segundo os valores-padrão do programa Praat, para a definição do início e do final das emissões.

Duas medidas do TMF foram obtidas: o TMF da vogal /a/ sustentada e a relação s/z. Ambas forneceram informações sobre a eficiência glótica dos falantes.

A diadococinesia oral de sílabas alternadas e em sequência foi utilizada na avaliação da velo- cidade dos movimentos articulatórios dos lábios e da língua. Os falantes foram instruídos a repetir cada sílaba (/pa/, /ta/ ou /ka/) ou a sequência de sílabas (/pataka/) o mais rápido possível, após inspiração profunda. Foram considerados os 5 primeiros segundos ou o segmento produzido no primeiro grupo respiratório (quando inferior a 5 segundos) e computado o número de sílabas produzidas, para o cálculo do número de sílabas por segundo.

A velocidade da fala foi mensurada a partir da leitura em voz alta de um texto com 129 palavras, em que o tempo em minutos foi registrado para o cálculo do número de palavras por minuto.

Este estudo foi aprovado pelo Comitê de Ética em Pesquisa da UNIFESP, sob número 0708/06, e ambos os participantes assinaram um termo de consentimento livre e esclarecido.

Os resultados obtidos na avaliação perceptivoauditiva e acústica de cada paciente serão apresentados de forma descritiva. Em relação aos parâmetros analisados perceptualmente, com exceção da inteligibilidade de fala, serão descritas as classificações e os valores referentes à intensidade do desvio encontrado. As medidas acústicas de F0 média, DP de FO e TMF ${ }^{9}$; jitter, shimmere PRH ${ }^{10}$; diadococinesia oral ${ }^{11}$; e velocidade de fala ${ }^{12}$, assim como os escores de inteligibilidade ${ }^{13}$ foram classificados com base nos critérios de normalidade propostos em diferentes estudos.

\section{RESULTADOS}

Na Tabela 1, são apresentados os resultados da avaliação perceptivo-auditiva da fala dos pacientes.

Os resultados da análise acústica são apresentados na Tabela 2. Foram fornecidos apenas os valores considerados desviados, segundo os critérios adotados.

\section{DISCUSSÃO}

Embora vários estudos tenham apontado a disartria como uma das primeiras manifestações neurológicas da DW ${ }^{14-16}$, poucos trabalhos propuseram-se a caracterizar as manifestações de fala presentes nesta doença 4,16,17.

Em pesquisa realizada com 20 sujeitos com DW, na qual 34 características de fala foram analisadas por meio de escala intervalar de 7 pontos, 17 delas foram consideradas proeminentes na doença 17. As manifestações proeminentes encontradas de acordo com a intensidade da alteração foram: ênfase reduzida, "bizarreness" (grau com que a fala desperta a atenção do ouvinte por ser incomum, peculiar ou bizarra), monopitch, monoloudness, consoantes imprecisas, inteligibilidade de fala alterada, velocidade de fala lenta, ênfase excessiva e 
equalizada, pitch grave, quebras articulatórias irregulares, hipernasalidade, pausas inapropriadas, voz rouca, prolongamento de fonemas, intervalos prolongados, voz tensa e frases curtas.

No presente estudo, dentre as manifestações comuns investigadas, constatou-se a presença de redução da inteligibilidade de fala, pitch grave, hipernasalidade, voz rouca e/ou tensa (Tabela 1), além de redução da velocidade de fala (Tabela 2). Contudo, outras características não consideradas proeminentes nos pacientes de estudo encontrado na literatura ${ }^{17}$ foram observadas nos sujeitos avaliados. Tais dados encontram-se na Tabela $1 \mathrm{e}$ foram: pitch agudo, instabilidade vocal, voz soprosa e ataque vocal aspirado. Exceto pelos desvios de pitch divergentes e pela presença de rouquidão no segundo caso, as manifestações citadas foram encontradas nos dois pacientes.

É importante salientar que a comparação dos achados entre tais estudos deve levar em consideração as diferenças metodológicas entre os mesmos, como o número de pacientes, os procedimentos de avaliação e os critérios de análise. No estudo mencionado ${ }^{17}$, foram categorizadas como proeminentes aquelas manifestações cuja média de classificação dos juízes para o grupo foi superior a 1,5.

Tabela 1 - Achados da avaliação perceptivo-auditiva da fala dos pacientes

\begin{tabular}{lcc}
\hline Parâmetros & Caso 1 & Caso 2 \\
\hline Tipo de voz & Pastosa (47) & Soprosa (80) \\
& Soprosa (37) & Rouca (59) \\
& Tensa (34) & Tensa (45) \\
Loudness & Áspera (27) & \\
Pitch & Reduzida (31) & Reduzida (48) \\
Ataque vocal & Agudo (40) & Grave (34) \\
Estabilidade vocal & Aspirado (23) & Aspirado (55) \\
Ressonância & Instável (50) & Instável (44) \\
Inteligibilidade da fala (\%) & Hipernasal (58) & Hipernasal (86) \\
Frases & & \\
Palavras & Reduzida (92) & Reduzida (3) \\
\hline
\end{tabular}

Tabela 2 - Achados da análise acústica da fala dos pacientes

\begin{tabular}{lcc}
\hline Parâmetros & Caso 1 & Caso 2 \\
\hline F0 (Hz) & Adequada & Adequada \\
Desvio padrão (Hz) & Adequado & Adequado \\
Jitter (\%) & Adequado & Aumentado $(0,77)$ \\
Shimmer (\%) & Aumentado $(8,57)$ & Aumentado $(14,31)$ \\
PRH & Aumentada $(0,082)$ & Aumentada $(0,817)$ \\
Tempo máximo de fonação & & \\
Vogal /a/ (segundos) & & \\
relação s/z & Reduzido $(5)$ & Reduzido $(8)$ \\
& Aumentada $(1,6)$ & \\
Diadococinesia oral & & \\
(sílabas/segundo) & & Reduzida $(2,60)$ \\
/pa/ & & Reduzida $(2,41)$ \\
/ta/ & Reduzida $(2,40)$ & Reduzida $(2,27)$ \\
/ka/ & Reduzida $(1,80)$ & Reduzida $(2,70)$ \\
/pataka/ & Reduzida $(1,93)$ & Reduzida $(59,53)$ \\
Velocidade da fala & Reduzida $(3,00)$ & \\
(palavras/minuto) & Reduzida $(99,30)$ & \\
\hline
\end{tabular}


Portanto, é possível que dentre as características não proeminentes estejam incluídas manifestações encontradas na maioria dos pacientes, porém com grau de alteração discreto.

Em pesquisa nacional, realizada com 42 disártricos de etiologias distintas, constatou-se que a voz rouca, soprosa e rouco-soprosa foram os tipos de voz mais frequentes, com a voz soprosa emergindo como um dos tipos mais comuns tanto na disartria hipocinética, quanto na espástica ${ }^{7}$. Similarmente, ambos os sujeitos de nosso estudo apresentaram soprosidade como uma característica de suas qualidades vocais.

Em concordância com os dois casos investigados, a ocorrência de loudness reduzida também foi descrita em outros dois estudos que avaliaram pacientes disártricos com DW ${ }^{4,16}$.

Com relação à ressonância, pesquisas realizadas com grupos de disártricos no Brasil indicaram a hipernasalidade ${ }^{18}$ ou a ressonância laringo-faríngea ${ }^{8}$ como a alteração de ressonância mais frequente nesses pacientes.

A redução da inteligibilidade de palavras e frases em falantes disártricos, mensurada através do método de transcrição ortográfica, também foi relatada por outros autores ${ }^{19}$. O grupo investigado incluía 13 disártricos com etiologias variadas, com diagnósticos de disartria do tipo mista, espástica ou atáxica.

Quanto aos dados da análise acústica observados na Tabela 2, além da redução da velocidade de fala já mencionada, constatou-se aumento de shimmer e PRH e redução do TMF e da diadococinesia oral em ambos os casos. Em outros estudos envolvendo pacientes disártricos com diferentes tipos de disartria, desvios de jitter e shimmer ${ }^{6-8}$, além de alteração das medidas de ruído e TMF foram encontrados 7,8 , assim como redução da diadococinesia oral ${ }^{11,20}$ e da velocidade de fala ${ }^{12}$.

Ainda que os pacientes do presente estudo tivessem diagnósticos sindrômicos e períodos de duração da doença distintos, foi possível identificar características comuns entre eles, indicando que essas podem constituir-se em traços característicos da fala de pacientes com disartria associada à DW. Todavia, diferenças também foram evidenciadas, as quais podem ser justificadas pelo tempo de evolução da doença, como será discutido a seguir.

O tempo de evolução da doença entre os sujeitos diferiu em, aproximadamente, 6 anos, com maior duração desta no segundo caso. Esse paciente demonstrou maior comprometimento da fala segundo a maioria dos parâmetros avaliados. Tal dado é compatível com seu diagnóstico sindrômico, uma vez que na disartria mista um comprometimento neurológico maior é habitualmente esperado, resultante de lesões que acometem várias estruturas do sistema nervoso.

No estudo que envolveu 20 pacientes com DW, dos 17 sujeitos com alterações de fala, todos apresentaram disartria mista, com componentes atáxico, espástico e hipocinético. Os autores encontraram que a combinação e a gravidade dos sintomas variaram entre os pacientes, as quais se relacionavam também com o curso da doença. $O$ tempo de duração médio da doença nesse grupo foi de 5,4 anos (DP=5,6) ${ }^{17}$.

Quanto à análise perceptivo-auditiva (Tabela 1), constatou-se que, para a maioria dos parâmetros avaliados (tipo de voz, loudness, ataque vocal, ressonância e inteligibilidade de fala), alterações mais intensas foram observadas no caso com o início do quadro há mais tempo, com diferenças superiores a 17 pontos na EAV. Quanto aos escores de inteligibilidade de fala, a diferença encontrada entre as pacientes alcançou valores de aproximadamente 55 desvios-padrão da média esperada na população normal ${ }^{13}$.

Em relação à acústica (Tabela 2), as medidas objetivas de jitter, shimmer, $\mathrm{PRH}$, diadococinesia oral de sílabas em seqüência e velocidade de fala revelaram maior desvio ou presença de alteração (jitter) no segundo caso. Nas medidas de shimmer, $\mathrm{PRH}$ e velocidade de fala, foram observadas diferenças de pelo menos 2 desvios-padrão da média esperada na população sem distúrbios da fala ${ }^{10,12}$. Já os valores de diadococinesia oral de sílabas em seqüência diferiram em menor magnitude entre os casos (0,4 a 1,1 desvios-padrão) ${ }^{11}$.

Sendo assim, os parâmetros tipo de voz, loudness, ataque vocal, ressonância, diadococinesia oral de sílabas em sequência e, particularmente, inteligibilidade de fala, jitter, shimmer, $\mathrm{PRH}$ e velocidade de fala (com maior magnitude das diferenças) aparentam serem mais sensíveis que os demais às mudanças na fala que acompanham a evolução da DW.

Entretanto, não se pode descartar a possibilidade de que a sensibilidade desses parâmetros à evolução da DW tenha sido influenciada também pela expressão da doença em cada indivíduo. Independentemente do padrão geral de comprometimento da fala apresentado pelo paciente, podemse encontrar alguns aspectos de sua produção mais alterados que outros, os quais podem variar para cada falante disártrico. Porém, desconsiderando os aspectos com resultados adequados em ambos os casos, notamos que o paciente com os sinais da doença há mais tempo apresentou comprometimento mais acentuado em $71 \%$ e $62 \%$ dos 
parâmetros da avaliação perceptual e acústica, respectivamente.

Os demais aspectos que se mostraram alterados com maior intensidade (pitch, estabilidade vocal, TMF e diadococinesia oral de sílabas alternadas) ou apenas desviados (relação $\mathrm{s} / \mathrm{z}$ ) no caso com menor duração do quadro podem revelar as diferenças individuais na expressão da doença. A restrita magnitude das diferenças encontradas entre esses parâmetros corrobora esta hipótese. Diferenças de apenas 6 pontos na EAV foram obtidas para os desvios de pitch e estabilidade vocal. $\mathrm{Na}$ diadococinesia oral de sílabas alternadas, tal diferença alcançou 0,4 desvio-padrão ${ }^{11}$.

Estudos prospectivos que envolvam maior número de falantes disártricos com DW precisam ser realizados, com o controle da variável tempo de evolução da doença, para que possam ser identificados com maior clareza os parâmetros de produção da fala mais sensíveis a sua identificação e à progressão da doença. Além disso, uma definição mais precisa das manifestações de fala características desses pacientes poderia ser útil ao diagnóstico precoce da doença. Nesse sentido, a ampliação dos parâmetros investigados seria imprescindível, abrangendo de forma mais homogênea todos os cinco subsistemas de produção da fala (respiração, fonação, ressonância, articulação e prosódia).

Os resultados de pesquisas com tal direcionamento poderiam ter grande aplicabilidade na clínica, com fins de diagnóstico precoce, avaliação da eficiência e eficácia de determinadas abordagens de tratamento, assim como para o acompanhamento desses pacientes.

\section{CONCLUSÃO}

A fala de ambos os pacientes foi caracterizada por: redução do TMF, voz soprosa e tensa, ataques vocais aspirados, instabilidade vocal, aumento de shimmer e PRH, hipernasalidade, redução da diadococinesia oral, da inteligibilidade e da velocidade da fala. Os resultados obtidos sugeriram que o paciente que cursa com a DW há mais tempo, apresentou prejuízo mais acentuado na maioria dos parâmetros analisados, tanto na avaliação perceptivo-auditiva quanto na análise acústica. Desta forma, são observadas diferenças relevantes nos dois casos. $O$ estudo sugere que as análises perceptual auditiva e acústica podem ser úteis no diagnóstico e acompanhamento longitudinal das disartrias, sobretudo nas doenças evolutivas.

\begin{abstract}
Background: dysarthria in Wilson's disease. Procedures: case study of two patients with disease's diagnosis, both young female adults, with six years of schooling. Perceptual analysis was done by four speech therapists. The following parameters were assessed: voice quality, loudness, pitch, vocal attack, vocal stability and resonance, as well as the degree of deviated parameters, measured by a visual analogue scale. In addition, the intelligibility was analysed by an orthographic transcription method. Praat 4.4.13 software was used for acoustic analysis. Fundamental frequency, phonatory stability, noise and temporal measures were equally assessed. Results: both patients have a speech characterized by: maximum phonation time reduced, breathiness and strained harsh voice quality, voice stoppages, transient breathiness, increased shimmer and noise-to-harmonics ratio, hypernasality. Oral diadochokinesia, speech rate and intelligibility of speech were reduced. The results suggested that the patient who showed Wilson's disease for longer time showed more acute impairments in most of the analyzed perceptual and acoustic parameters. Conclusion: both cases showed similar caracteristics. These features seem to be typical in dysarthric patients with Wilson's disease. However, differences were also observed, and can be related to the disease's evolution.
\end{abstract}

KEYWORDS: Dysarthria; Hepatolenticular Degeneration; Speech Production Measurement; Speech Acoustics 


\section{REFERÊNCIAS}

1. Wilson SAK. Progressive lenticular degeneration: a familial nervous disease associated with cirrhosis of the liver. Brain. 1912; 34:295-509.

2. Hoogenraad TU. Paradigm shift in treatment of Wilson's disease: zinc therapy now treatment of choice. Brain Develop. 2006; 28(3):141-6.

3. Sinha S, Taly AB. Withdrawal of penicillamine from zinc sulphate-penicillamine maintenance therapy in Wilson's disease: promising, safe and cheap. J Neurol Sci. 2008; 264(1-2):129-32.

4. Rahman S, Siddiqui NI, Paul GK, Sarker CB, Rahman KM. A case report on Wilson's disease. Mymensingh Med J. 2003 Jul; 12(2):142-5.

5. Darley FL, Aronson AE, Brown JR. Differential diagnostic patterns of dysarthria. J Speech Hear Res. 1969; 12(2):246-69.

6. Amir O, Dukas M, Shnaps-Baum R. The effect of a 'voice course' on the voices of people with and without pathologies: preliminary observations. Logoped Phoniatr Vocol. 2005; 30(2):63-71.

7. Carrillo L, Ortiz KZ. Análise vocal (auditiva e acústica) nas disartrias. Pró-Fono. 2007; 19(4):381-6.

8. Ortiz KZ, Carrillo L. Comparação entre as análises auditiva e acústica nas disartrias. Rev Soc Bras Fonoaudiol. 2008; 13(4):325-31.

9. Behlau M, Madazio G, Feijó D, Pontes P. Avaliação de voz. In: Behlau M. Voz: o livro do especialista. Rio de Janeiro: Revinter. 2001. p. 83-245.

10. Oguz H, Demirci M, Safak MA, Arslan N, Islam A, Kargin S. Effects of unilateral vocal cord paralysis on objective voice measures obtained by Praat. Eur Arch Otorhinolaryngol. 2007; 264(3):257-61.
11. Depret MMP. Análise da diadococinesia articulatória e laríngea em indivíduos com e sem transtornos neurológicos [dissertação]. São Paulo (SP): Universidade Federal de São Paulo; 2005.

12. Oliveira CR, Ortiz KZ, Vieira MM. Disartria: estudo da velocidade de fala. Pró-Fono. 2004; 16(1):39-48.

13. Barreto SS, Ortiz KZ. Influência da velocidade articulatória e da intensidade na inteligibilidade de fala. Pró-Fono. 2008; 20(2):87-92.

14. Machado AA, Deguti MM, Genschel J, Cançado EL, Bochow B, Schmidt $H$, et al. Neurological manifestations and ATP7B mutations in Wilson's disease. Parkinsonism Relat Disord. 2008; 14(3):246-9.

15. Carlson MD, Al-Mateen M, Brewer GJ. Atypical childhood Wilson's disease. Pediatr Neurol. 2004; 30(1):57-60.

16. Bono W, Moutie O, Benomar A, Aïdi S, El Alaoui-Faris M, Yahyaoui M, et al. La maladie de Wilson. Étude clinique, thérapeutique et évolutive de 21 Cas. Rev Méd Interne. 2002; 23(5):419-31.

17. Berry WR, Darley FL, Aronson AE, Goldstein NP. Dysarthria in Wilson's Disease. J Speech Hear Res. 1974; 17(2):169-83.

18. Miranda CS, Soares ECS, Ortiz KZ. Eficácia do processo terapêutico fonoaudiológico em grupo para disartria. Fono Atual. 2005; 8(32):32-9.

19. Yorkston KM, Beukelman DR. Communication efficiency of dysarthric speakers as measured by sentence intelligibility and speaking rate. J Speech Hear Disord. 1981; 46:296-301.

20. Wang YT, Kent RD, Duffy JR, Thomas JE, Weismer G. Alternating motion rate as an index of speech motor disroder in traumatic brain injury. Clin Ling Phonetics. 2004; 18(1):57-84.

RECEBIDO EM: 27/11/2008

ACEITO EM: 06/04/2009

Endereço para correspondência:

Natália Casagrande Brabo

Rua Machado, 144

Cerquilho - SP

CEP: $18520-000$

E-mail: nataliacasagrande@ @otmail.com 